\title{
Peripheral NMDA Receptors Mediate Antidromic Nerve Stimulation-Induced Tactile Hypersensitivity in the Rat
}

\author{
Jun Ho Jang, ${ }^{1}$ Taick Sang Nam,,3 Jaebeom Jun,, ${ }^{2,4}$ Se Jung Jung, \\ Dong-Wook Kim, ${ }^{2,4}$ and Joong Woo Leem ${ }^{2,3,5}$ \\ ${ }^{1}$ Dental Research Institute, Seoul National University, Seoul 110-744, Republic of Korea \\ ${ }^{2}$ Department of Physiology, Yonsei University College of Medicine, Seoul 120-752, Republic of Korea \\ ${ }^{3}$ Brain Research Institute, Yonsei University College of Medicine, Seoul 120-752, Republic of Korea \\ ${ }^{4}$ Brain Korea 21 PLUS Project for Medical Science, Yonsei University College of Medicine, Seoul 120-752, Republic of Korea \\ ${ }^{5}$ Graduate Program in Cognitive Science Yonsei University, Seoul 120-752, Republic of Korea
}

Correspondence should be addressed to Joong Woo Leem; jwleem@yuhs.ac

Received 19 August 2015; Accepted 19 November 2015

Academic Editor: Seungkyu Lee

Copyright (C) 2015 Jun Ho Jang et al. This is an open access article distributed under the Creative Commons Attribution License, which permits unrestricted use, distribution, and reproduction in any medium, provided the original work is properly cited.

\begin{abstract}
We investigated the role of peripheral NMDA receptors (NMDARs) in antidromic nerve stimulation-induced tactile hypersensitivity outside the skin area innervated by stimulated nerve. Tetanic electrical stimulation (ES) of the decentralized L5 spinal nerve, which induced enlargement of plasma extravasation, resulted in tactile hypersensitivity in the L4 plantar dermatome of the hindpaw. When intraplantar (i.pl.) injection was administered into the L4 dermatome before ES, NMDAR and group-I metabotropic Glu receptor (mGluR) antagonists and group-II mGluR agonist but not AMPA/kainate receptor antagonist prevented ES-induced hypersensitivity. I.pl. injection of PKA or PKC inhibitors also prevented ES-induced hypersensitivity. When the same injections were administered after establishment of ES-induced hypersensitivity, hypersensitivity was partially reduced by NMDAR antagonist only. In naïve animals, i.pl. Glu injection into the L4 dermatome induced tactile hypersensitivity, which was blocked by NMDAR antagonist and PKA and PKC inhibitors. These results suggest that the peripheral release of Glu, induced by antidromic nerve stimulation, leads to the expansion of tactile hypersensitive skin probably via nociceptor sensitization spread due to the diffusion of Glu into the skin near the release site. In addition, intracellular PKA- and PKC-dependent mechanisms mediated mainly by NMDAR activation are involved in Glu-induced nociceptor sensitization and subsequent hypersensitivity.
\end{abstract}

\section{Introduction}

Primary afferent nociceptors are responsible for converting harmful stimuli in the peripheral tissue into internal electrical impulses and then conveying these impulses to the central nervous system for pain perception. In addition to their afferent functions, evidence indicates that nociceptors also have local efferent functions. Upon activation, nociceptive afferents, especially the unmyelinated C-fibers that constitute the largest proportion of cutaneous nociceptive afferents, are able to provoke the local release of various algesic substances at the peripheral terminals [1-3]. Indeed, stimulation of C-fibers results in a local increase in levels of glutamate (Glu), substance P (SP), and calcitonin gene-related peptide
(CGRP) [4-7]. Peripherally released algesic substances are known to cause neurogenic inflammation $[1,8]$ and are also likely to diffuse to receptors expressed on nearby nociceptors in a paracrine manner to modulate neuronal excitability.

There is significant evidence for the modulatory role of Glu in peripheral nociception. Both ionotropic and metabotropic Glu receptors (mGluRs) are present in the peripheral terminals of unmyelinated afferents [9-13]. The subcutaneous injection of Glu and specific GluR agonists produces pain behaviors that are blocked by their corresponding antagonists [10, 14]. Furthermore, local injection of GluR antagonists attenuates pain behaviors in various experimental models of pain $[9,15-24]$. In addition, peripheral Glu is able to increase the excitability of nociceptors under both normal 
and pathological conditions [21, 25]. Glu-induced tactile hyperalgesia is mediated by intracellular signaling pathways that activate protein kinases such as protein kinases A (PKA) and $\mathrm{C}(\mathrm{PKC})$ in the primary sensory neurons $[26,27]$.

In a previous study using rats with an injury to the lumbar L5 spinal nerve (L5 SN) that had previously undergone a L5 dorsal root rhizotomy (DR) (decentralized L5 $\mathrm{SN}$ ), we proposed Wallerian degeneration and peripherally propagating injury discharge induced by nerve ligation/cut as peripheral contributions to nerve injury-induced neuropathic pain [28]. The present study was performed using the same rhizotomized rats to investigate the role of peripherally propagating nerve impulses, which were evoked by a tetanic electrical stimulation (ES) that was revealed to release Glu from peripheral terminals of primary afferent fibers [4], in the development of hypersensitivity outside the skin area innervated by the stimulated nerve. To achieve this goal, we examined whether antidromic stimulation of the decentralized L5 SN induces tactile hypersensitivity on the L4 plantar dermatome of the hind-paw. In addition, we explored which subtypes of GluRs mediate such hypersensitivity. We also examined whether PKA and PKC were involved in this hypersensitivity.

\section{Materials and Methods}

2.1. Experimental Animals and Surgical Procedures. Adult male rats (150-250 g; Harlan Sprague-Dawley, Koatech Co., Gyeonggi-do, Korea) were used. The animals were housed in groups of three to four with food and water available ad libitum. All animals were acclimated to a 12-12 lightdark cycle for approximately 1 week before the surgery and behavioral testing. All the experiments were conducted in accordance with the approval of the Institutional Animal Care and Use Committee of Yonsei University, Seoul, Korea.

All the surgical procedures were conducted under enflurane anesthesia (2-3\% enflurane- $\mathrm{O}_{2}$ mixture). The animals were initially subjected to an L5 DR before receiving subsequent manipulations. For the L5 DR, a longitudinal skin incision was made to expose the L3-L6 vertebral segments, and a hemilaminectomy was performed at the left L5 segments. Care was taken to avoid any mechanical trauma to the spinal cord, the dorsal roots, and the dorsal root ganglion (DRG). The dura mater was opened, and the left L5 dorsal root was exposed and sectioned $2-3 \mathrm{~mm}$ proximal to the L5 DRG. A small portion of the distal end of the dissected root was sectioned for removal.

2.2. Electrical Stimulation. A tetanic ES of the L5 SN was conducted in rats that received a left L5 DR (Figure 1(a)). In these rats, if mild tactile hyperalgesia had been present, then it had worn off within a week after the rhizotomy. The animals were under enflurane anesthesia throughout the ES experiment. After removing the left transverse process of the L6 vertebra, the left L5 SN was exposed. A piece of parafilm was placed underneath the exposed $\mathrm{SN}$ to isolate it from the surrounding tissue. A pair of thin flexible silver wires (0.005 in diameter) was placed $4-6 \mathrm{~mm}$ distal to the L5 DRG to gently loop around the L5 SN (3 mm apart). The other ends of the wires were connected to a stimulus isolation unit (SI1850, World Precision Instruments, New Haven, CT, USA). Square-wave pulses $(0.5 \mathrm{~ms}, 4 \mathrm{~Hz})$ were applied with graded levels of current to determine the lowest level of current needed to elicit a muscle twitch or the threshold current. The current pulses at strengths 200 times the threshold current $(2-4 \mathrm{~mA}, 200 \times \mathrm{TH})$ were delivered for $5 \mathrm{~min}$ to activate both the A- and C-fibers in the L5 SN. For the sham-operated group, all procedures were conducted in exactly the same way as for the ES-treated animals, except that no ES was delivered.

The plasma extravasation, which is one of the characteristic symptoms of neurogenic inflammation, was induced by the ES of the L5 SN using extra animals that received an intravenous injection of Evans blue dye (Sigma, St. Louis, MO, USA) in the lateral tail vein $(30 \mathrm{mg} / \mathrm{kg}, 2 \%$ solution) before the ES. The images of dye extravasation seen as blue on the plantar surface were captured with a digital video camera to determine whether changes in the extravasation area occurred during the ES.

\subsection{Drug Preparation and Administration. Noncompetitive} NMDA ( $N$-methyl-D-aspartate) receptor (NMDAR) antagonist MK-801 and competitive AMPA (amino-3-hydroxy-5methylisoxazole-4-propionic acid)/kainate receptor antagonist NBQX (2,3-dioxo-6-nitro-1,2,3,4,-tetrahydrobenzo[f] quinoxaline-7-sulfonamide) were dissolved in phosphate-buffered saline (PBS), $\mathrm{pH}$ 7.4. A competitive group-I mGluR antagonist DL-AP3 (DL-amino-3-phosphonopropionic acid) and selective group-II mGluR agonist APDC $((2 \mathrm{R}, 4 \mathrm{R})-4$ aminopyrrolidine-2,4-dicarboxylate) were first prepared as a stock solution in $100 \mathrm{mM} \mathrm{NaOH}$ and then diluted to final concentrations with PBS. For PKA and PKC inhibition, staurosporine (a broad-spectrum kinase inhibitor) and calphostin C (a selective PKC inhibitor) were first prepared as a stock solution in $1 \mathrm{mM} \mathrm{DMSO}$ and then diluted to the final concentration with PBS. The Glu and H-89 (a selective PKA inhibitor) were dissolved in PBS. All the drugs were purchased from Tocris Cookson (Bristol, UK). Corresponding vehicles were prepared in an identical manner without the addition of the drug. The selection of drug doses used in this study was based on our previous study in which the effective doses of drugs without obvious side effects have been chosen [15]. These drug concentrations were lower than those used by other groups for various experiments, excluding the possibility of off-target effects [16-18, 30, 31].

Under enflurane anesthesia (3\%, 2-3 min), $30 \mu \mathrm{L}$ of the drugs or corresponding vehicles was injected subcutaneously under the hind-paw skin using a $50 \mu \mathrm{L}$ Hamilton syringe (Reno, NV, USA) with a 28 -gauge needle. For this intraplantar (i.pl.) injection, the needle was inserted into the skin in the middle of the plantar surface proximal to the proximal tori of the hind-paw and advanced approximately $10 \mathrm{~mm}$ so that it reached the center of the circle surrounded by the tori, where the solution was injected and formed a bleb that disappeared within $10 \mathrm{~min}$ (Figure 1(b)). Pretreatments were performed immediately (i.e., within $10 \mathrm{~min}$ ) prior to the ES, and posttreatments were performed 3 days after the ES. To 


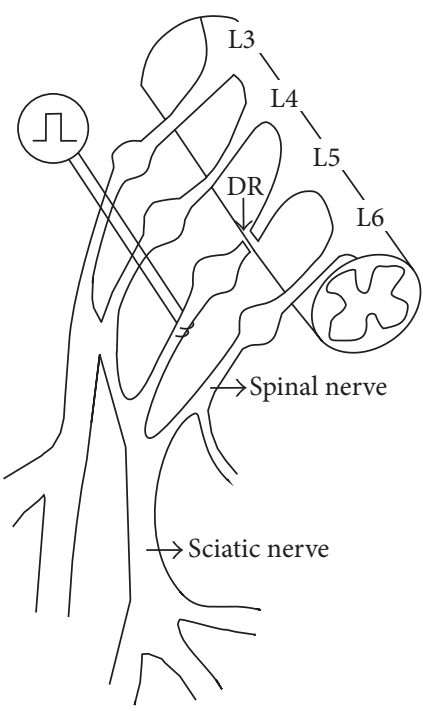

(a)

Before ES

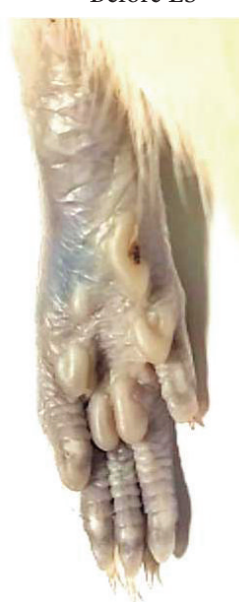

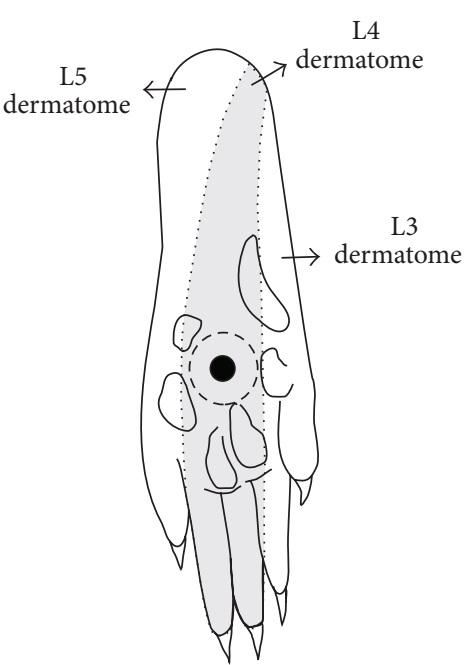

(b)

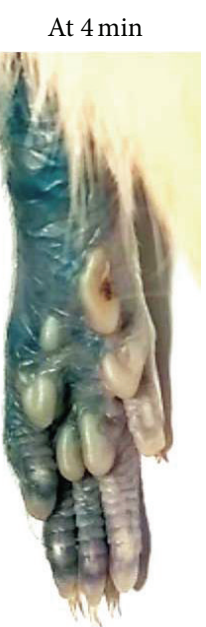

(c)

FIGURE 1: The locations of electrical stimulation (ES), intraplantar (i.pl.) injection of substances, and paw sensitivity testing and an example of ES-induced plasma extravasation. (a) An L5 spinal nerve (SN) was decentralized by L5 dorsal rhizotomy (DR). A high-level tetanic ES (2-4 mA, $0.5 \mathrm{~ms}$ pulse, $4 \mathrm{~Hz}$, and $5 \mathrm{~min}$ ) was applied to the decentralized L5 SN 4-6 mm distal to the L5 dorsal root ganglion (DRG). (b) The tactile sensitivity testing was performed in an area (marked by a filled circle) located in the center of the circle surrounded by the tori, which is almost matched to the midpoint of the L4 plantar dermatome (shaded area) [29] of the hind-paw. A $30 \mu \mathrm{L}$ i.pl. injection of different substances was administered subcutaneously into the skin area subjected to tactile sensitivity testing, forming a bleb that disappeared within $10 \mathrm{~min}$ after the injection (bleb area marked by a dotted circle). (c) Following Evans blue injection (i.v., $30 \mathrm{mg} / \mathrm{kg}$, and $2 \%$ solution), areas of plasma extravasation induced by the ES of the L5 SN are seen as blue. Note that dye extravasation covered the L5 plantar dermatome within the first 90 seconds of the ES, and the extravasation area extended to the L4 plantar dermatome within 4 minutes of the ES.

assess the antinociceptive effects of PKA and PKC inhibitors on Glu-induced hypersensitivity in naïve rats, pretreatment was conducted 10 min before the Glu treatment with both i.pl. injections administered at the same site.

2.4. Behavioral Testing. Each rat was placed in a plexiglass cage $\left(8 \times 8 \times 20 \mathrm{~cm}^{3}\right)$ above a wire mesh bottom that allowed full access to their paws. Following a $15 \mathrm{~min}$ acclimation period, the rats underwent behavioral testing. Tactile hypersensitivity was evaluated by measuring the paw withdrawal threshold (PWT) upon the application of a von Frey filament using the Dixon's up-down testing paradigm [32]. A series of von Frey filaments, ranging from 0.3 to $15.0 \mathrm{~g}$, were applied perpendicular to the center of the circle surrounded by the tori of the hind-paw (avoiding the keratinized foot pads, see Figure 1(b)) for 2-3 s until each filament bent slightly, starting with a $2.5 \mathrm{~g}$ stimulus. For the PWT measurement, the $50 \%$ withdrawal threshold was determined based on an equation and the calibration table described by Chaplan et al. [33]. Behavioral testing was performed by investigators who 
were blind to the surgery and treatment that the animals had received.

2.5. Statistical Analysis. To determine the differences between the different treatment groups on a given testing day, the data were analyzed using a Mann-Whitney ranksum test or a Kruskal-Wallis analysis of variance (ANOVA) followed by Dunn's test for multiple comparisons. The differences from baseline within a given treatment group were analyzed using a Friedman repeated measures ANOVA followed by Dunn's test for multiple comparisons. $P<0.05$ was considered to be statistically significant. Data are represented as mean \pm SEM.

\section{Results}

3.1. Tetanic ES-Induced Tactile Hypersensitivity. We investigated using rats with L5 DR whether a high-level tetanic ES (2-4 mA, $0.5 \mathrm{~ms}$ pulse, $4 \mathrm{~Hz}$, and $5 \mathrm{~min}$ ) of the L5 SN (L5 SN-ES) induced tactile hypersensitivity on the L4 dermatome. The tactile sensitivity was tested by measuring PWT using von Frey filaments applied to the center of the hind-paw glabrous skin surrounded by the tori, which is almost matched to the midpoint of the L4 plantar dermatome (Figure 1). This L5 SN-ES was observed to induce neurogenic inflammation consistently in all test animals, as judged by extravasated Evans blue, which covered the L5 plantar dermatome within the first 90 seconds of the ES and extended to the L4 plantar dermatome within 4 minutes of the ES. No further enlargement of the extravasation zone was observed during the remaining ES period. A typical example of ESinduced plasma extravasation observed in five rats tested was illustrated in Figure 1(c).

As for PWT changes (Figure 2), L5 SN-ES resulted in a significant decrease in the PWTs of the L4 plantar dermatome of the hind-paw ipsilateral to the ES as compared with the baseline. This decrease in the PWTs lasted for 7 days from poststimulation days 1 through 7 . Such decreases also were significant in comparison with the PWTs of sham animals or the contralateral hind-paw.

3.2. Effects of Pretreatment in the Periphery with $M K-801$, NBQX, DL-AP3, or APDC on ES-Induced Tactile Hypersensitivity. The involvement of NMDA and AMPA/kainate receptors in the initiation of L5 SN-ES-induced tactile hypersensitivity of the L4 plantar dermatome was examined using rats with decentralized L5 SN. As seen in Figure 3(a), an i.pl. MK-801 (20 nM) injection up to $10 \mathrm{~min}$ prior to the ES into the midpoint of the L4 plantar dermatome of the hindpaw ipsilateral to the ES resulted in a significant increase in ES-induced decreased PWTs compared with vehicle-treated controls. These data indicate that NMDARs in the L4 plantar dermatome contribute to the initiation of L5 SN-ES-induced tactile hypersensitivity. Animals administered an i.pl. NBQX $(100 \mathrm{nM})$ injection prior to the ES exhibited no significant difference in ES-induced decreased PWTs compared with vehicle-treated controls (Figure 3(b)), which indicates that AMPA/kainate receptors in the L4 plantar dermatome are

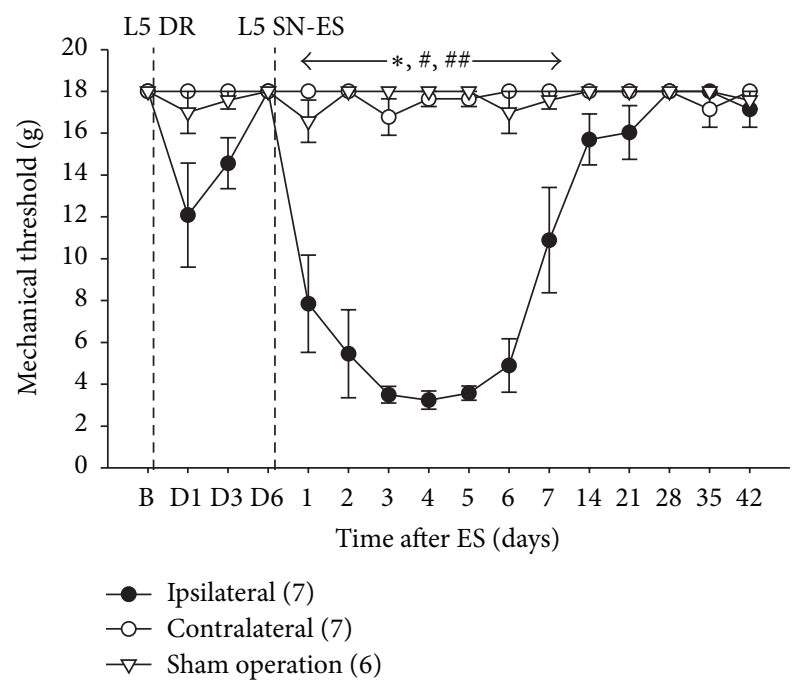

FIGURE 2: Tactile hypersensitivity following tetanic ES of the decentralized L5 SN. A tetanic ES of the L5 SN (L5 SN-ES) in rats with L5 DR induced a significant decrease in PWTs in the affected hind-paws on days 1 through 7 after ES compared with the baseline, contralateral hind-paws, and sham-operated animals. The abbreviations $\mathrm{B}$ and $\mathrm{D}$ denote the baseline before $\mathrm{DR}$ and postoperative days after DR, respectively. The figures in parentheses indicate the number of animals used. The error bars represent the SEM. ${ }^{*} P<0.05$ versus sham animals. ${ }^{\#} P<0.05$ versus baseline. ${ }^{\# \#} P<0.05$ versus contralateral hind-paws.

unlikely to contribute to the initiation of L5 SN-ES-induced hypersensitivity.

The involvement of mGluRs in the initiation of L5 SN-ES-induced tactile hypersensitivity of the L4 plantar dermatome was investigated. As seen in Figures 3(c) and 3(d), animals administered an i.pl. injection of a competitive group-I mGluR antagonist DL-AP3 $(70 \mathrm{nM})$ or a selective group-II mGluR agonist APDC $(20 \mathrm{nM})$ prior to the ES into the midpoint of the L4 plantar dermatome resulted in a significant increase in ES-induced decreased PWTs as compared with vehicle-treated controls. These data indicate that the activation of group-I mGluRs and the inhibition of group-II mGluRs in the L4 plantar dermatome are implicated in the initiation of L5 SN-ES-induced hypersensitivity.

3.3. Effects of Posttreatment in the Periphery with MK-801, NBQX, DL-AP3, or APDC on ES-Induced Tactile Hypersensitivity. The involvement of NMDA and AMPA/kainate receptors in the maintenance of $\mathrm{L} 5 \mathrm{SN}$-ES-induced tactile hypersensitivity of the L4 plantar dermatome was examined using rats with a decentralized L5 SN. As seen in Figure 4(a), an i.pl. injection of MK-801 (20 nM) into the midpoint of the L4 plantar dermatome on day 3 after ES resulted in a significant increase in ES-induced decreased PWTs at 15, 30 , and 45 min posttreatment compared with vehicle-treated controls, which indicates a contribution of NMDARs in the L4 plantar dermatome to the maintenance of L5 SNES-induced hypersensitivity. Animals administered an i.pl. injection of NBQX $(100 \mathrm{nM})$ into the midpoint of the L4 


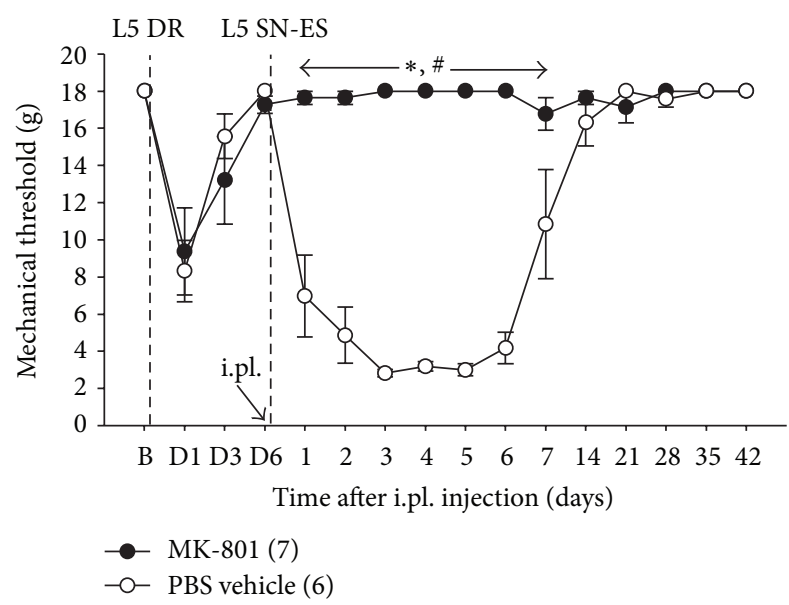

(a)

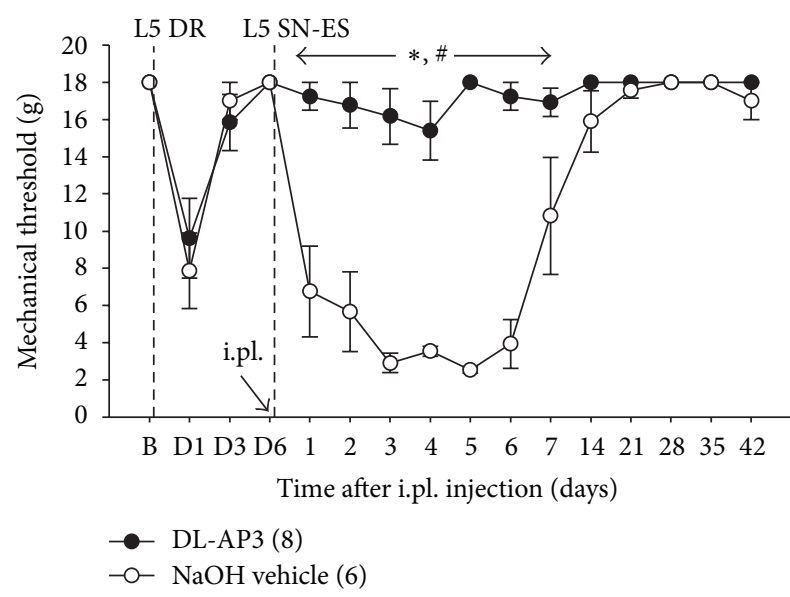

(c)

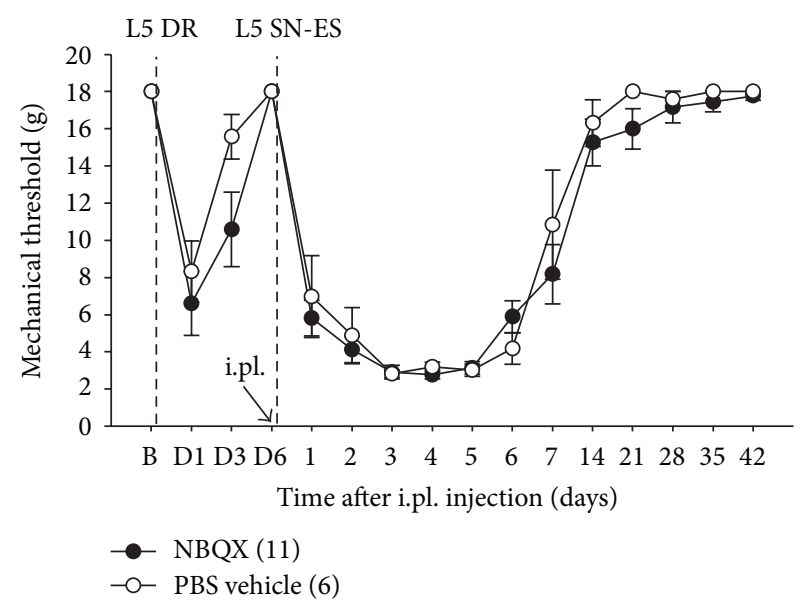

(b)

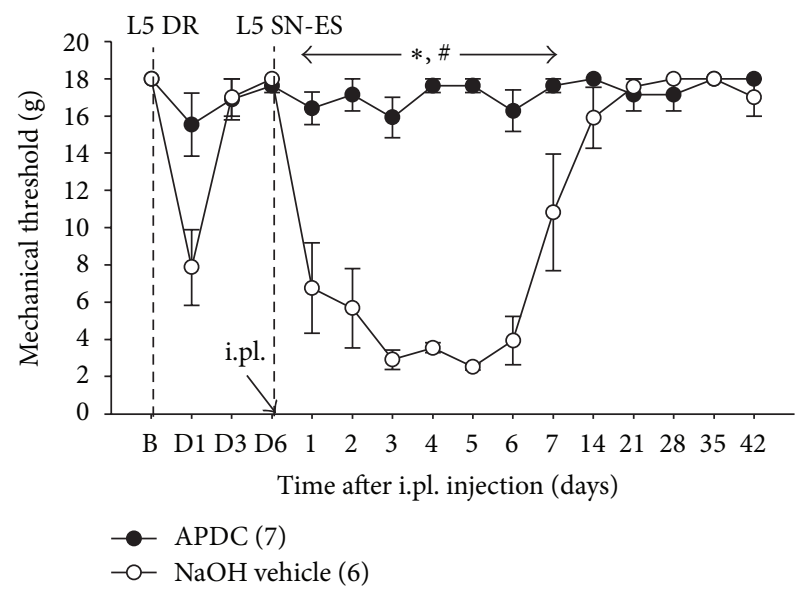

(d)

FIGURE 3: Effects of MK-801, NBQX, DL-AP3, or APDC pretreatment in the periphery on ES-induced PWT reduction. (a) In rats with L5 DR, an i.pl. injection of MK-801 $(20 \mathrm{nM})$ administered immediately before the ES of L5 SN (L5 SN-ES) into the midpoint of the L4 plantar dermatome resulted in a significant increase in ES-induced decreased PWTs compared with vehicle-treated controls. (b) An i.pl. NBQX $(100 \mathrm{nM})$ injection administered immediately prior to the ES produced no significant difference in ES-induced decreased PWTs from vehicle-treated controls. (c and d) Animals administered an i.pl. injection of DL-AP3 (70 nM) or APDC (20 nM) immediately prior to the ES exhibited a significant increase in ES-induced decreased PWTs compared with vehicle-treated controls. The abbreviations B and D are defined in Figure 2. The values in parentheses indicate the number of animals used. The error bars represent the SEM. ${ }^{*} P<0.05$ versus vehicle groups. ${ }^{\#} P<0.05$ versus baseline.

plantar dermatome on day 3 after ES exhibited no significant changes in ES-induced decreased PWTs compared with vehicle-treated controls (Figure 4(b)), which indicates no participation of AMPA/kainate receptors in the L4 plantar dermatome in maintaining L5 SN-ES-induced hypersensitivity.

The involvement of group-I and -II mGluRs in the maintenance of L5 SN-ES-induced tactile hypersensitivity was tested. As seen in Figures 4(c) and 4(d), animals administered an i.pl. injection of DL-AP3 $(70 \mathrm{nM})$ or APDC $(20 \mathrm{nM})$ into the midpoint of the L4 plantar dermatome on day 3 after ES exhibited no significant changes in ES-induced decreased PWTs compared with vehicle-treated controls. These data indicate that neither group-I mGluRs nor group-II mGluRs in the L4 plantar dermatome contribute to the maintenance of L5 SN-ES-induced hypersensitivity.
3.4. Effects of Pre- and Posttreatment in the Periphery with Staurosporine, Calphostin C, or H-89 on ES-Induced Tactile Hypersensitivity. The involvement of the intracellular signaling molecules PKA and PKC in the initiation of L5 SN-ESinduced tactile hypersensitivity was tested using rats with decentralized L5 SN. As seen in Figures 5(a), 5(b), and 5(c), an i.pl. injection of staurosporine (a broad-spectrum kinase inhibitor, $70 \mu \mathrm{M})$, calphostin $\mathrm{C}(12 \mu \mathrm{M})$, or $\mathrm{H}-89(10 \mu \mathrm{M})$ into the midpoint of the L4 plantar dermatome up to 10 min prior to the ES resulted in a significant increase in ES-induced decreased PWTs compared with vehicle-treated controls. These data indicate that both PKA and PKC participate in initiating L5 SN-ES-induced hypersensitivity in the L4 plantar dermatome.

When an i.pl. injection was administered on day 3 after ES into the midpoint of the L5 plantar dermatome, 


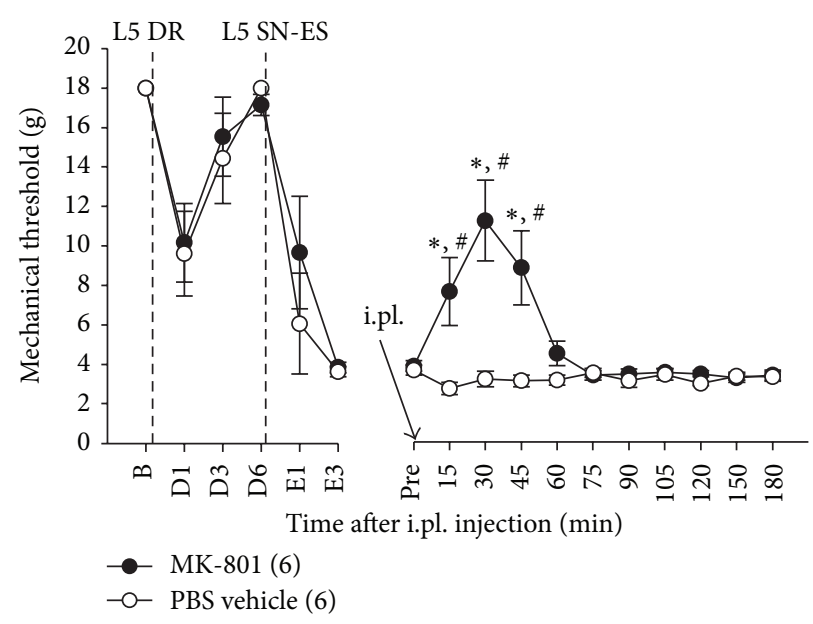

(a)

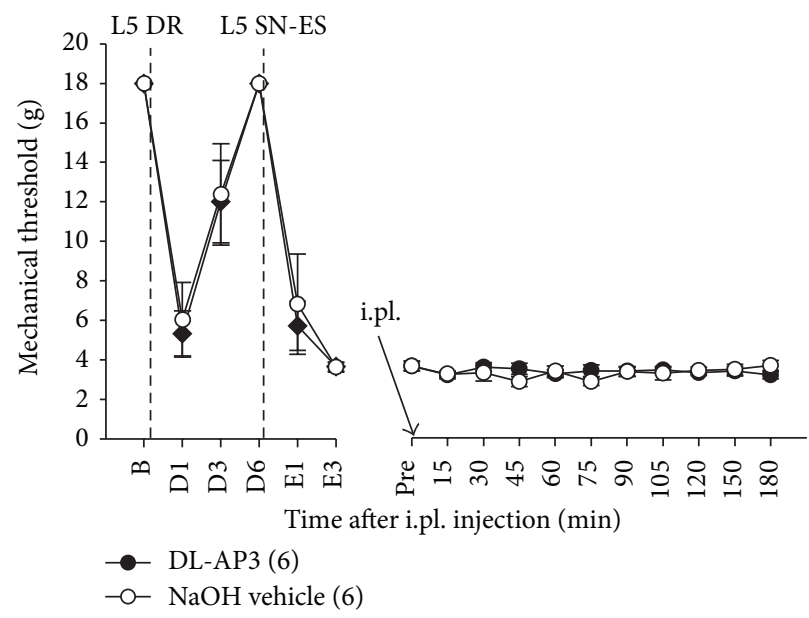

(c)

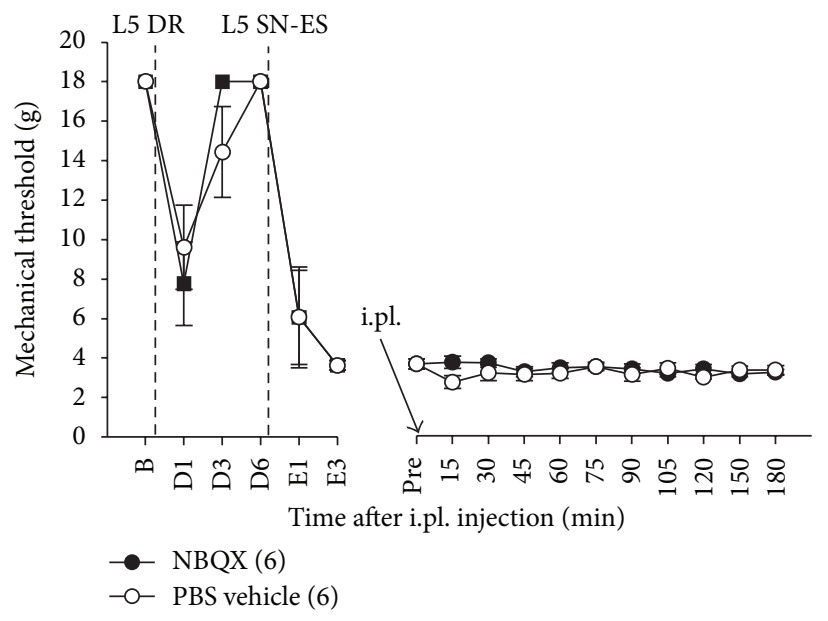

(b)

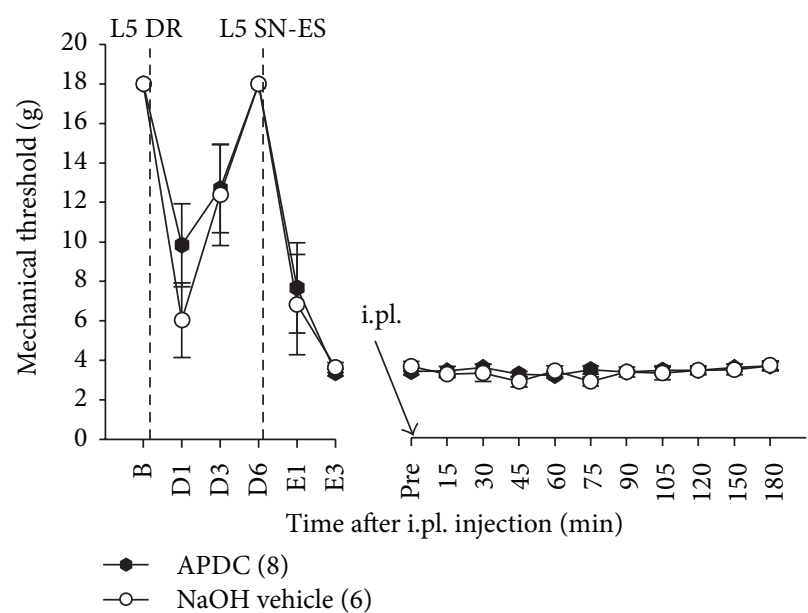

(d)

FIGURE 4: Effects of MK-801, NBQX, DL-AP3, or APDC posttreatment in the periphery on ES-induced PWT reduction. (a) In rats with L5 DR, an i.pl. injection of MK-801 (20 nM) administered 3 days after the ES of L5 SN (L5 SN-ES) into the midpoint of the L4 plantar dermatome resulted in a significant increase in ES-induced decreased PWTs at 15, 30, and 45 min after treatment compared with vehicle-treated controls. (b-d) No differences in ES-induced decreased PWTs were observed between animals treated with NBQX (100 nM), DL-AP3 (70 nM), or APDC (20 nM) and vehicle-treated controls. The abbreviations B, D, E, and Pre denote the baseline before DR, postoperative days after DR, postoperative days after ES, and baseline prior to i.pl. injection, respectively. The values in parentheses indicate the number of animals used. The error bars represent the SEM. ${ }^{*} P<0.05$ versus vehicle group. ${ }^{\#} P<0.05$ versus baseline.

animals treated with staurosporine $(70 \mu \mathrm{M})$, calphostin $\mathrm{C}$ $(12 \mu \mathrm{M})$, or $\mathrm{H}-89(10 \mu \mathrm{M})$ exhibited no significant changes in ES-induced decreased PWTs compared with vehicletreated controls (Figures 6(a), 6(b), and 6(c)). No effects were observed at higher doses of each drug. Thus, neither PKA nor PKC are implicated in maintaining L5 SN-ES-induced hypersensitivity in the L4 plantar dermatome.

3.5. Effects of Pretreatment in the Periphery with MK-801, Calphostin C, or H-89 on Glu-Induced Tactile Hypersensitivity. The involvement of NMDAR, PKA, and PKC in initiation of Glu-induced tactile hypersensitivity was investigated using naïve rats. As seen in Figure 7, an i.pl. injection of Glu into the midpoint of the L4 plantar dermatome of the hind-paw in naïve rats resulted in a significant decrease in PWTs, which lasted approximately $6 \mathrm{~h}$ compared with the baseline. An i.pl. injection of MK-801 (20 nM), calphostin C (12 $\mu \mathrm{M})$, or $\mathrm{H}-89(10 \mu \mathrm{M})$ into the same site $10 \mathrm{~min}$ prior to i.pl. Glu injection produced a significant increase in Glu-induced decreased PWTs compared with vehicle-pretreated controls. These data indicate that Glu induces tactile hypersensitivity in the L4 plantar dermatome, and this hypersensitivity is likely mediated by activation of NMDARs through intracellular PKA and PKC signaling pathways.

\section{Discussion}

4.1. Tactile Hypersensitivity Induced by the Antidromic ES of the Decentralized L5 SN. The efferent function of nociceptors can be achieved via the release of algesic substances from 


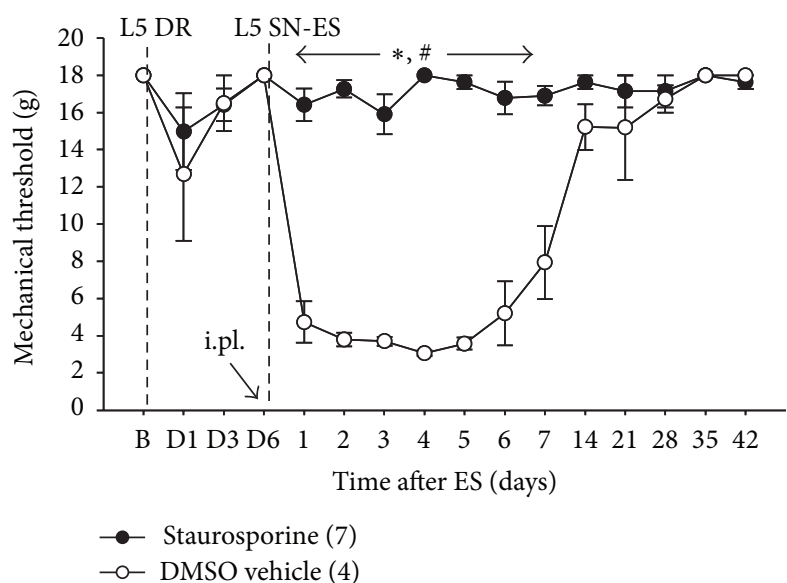

(a)

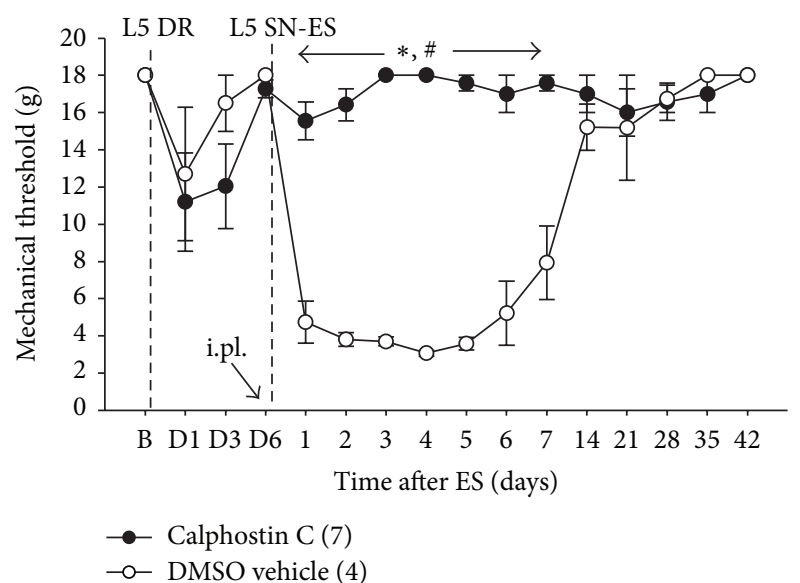

(b)

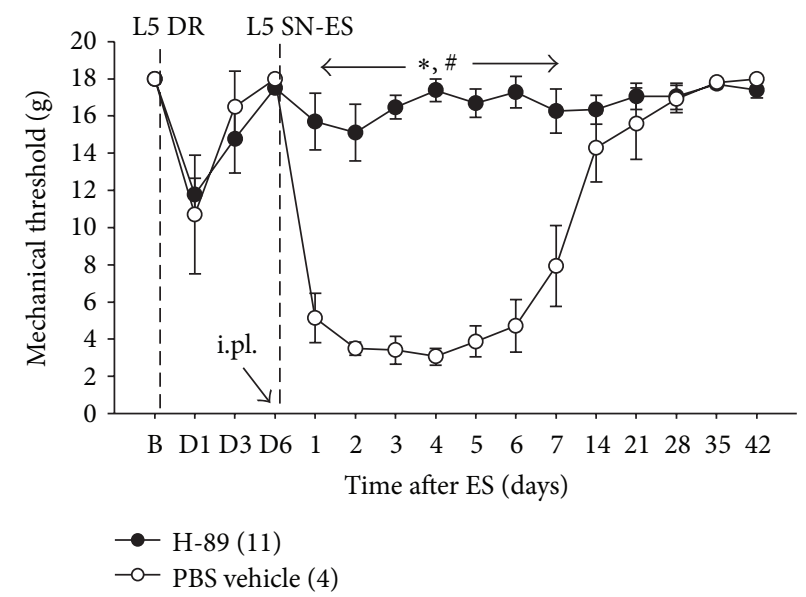

(c)

FIGURE 5: Effects of staurosporine, calphostin C, or H-89 pretreatment in the periphery on ES-induced PWT reduction. (a-c) In rats with L5 DR, an i.pl. injection of staurosporine $(70 \mu \mathrm{M})$, calphostin C $(12 \mu \mathrm{M})$, or H-89 $(10 \mu \mathrm{M})$ administered immediately prior to the ES of L5 SN (L5 SN-ES) into the midpoint of the L4 plantar dermatome resulted in a significant increase in ES-induced decreased PWTs compared with vehicle-treated controls. The abbreviations B and D are defined in Figure 2. The values in parentheses indicate the number of animals used. The error bars represent the SEM. ${ }^{*} P<0.05$ versus vehicle group. ${ }^{\#} P<0.05$ versus baseline.

their peripheral terminals by noxious stimulation [2] and also by the antidromic propagation of impulses, for example, peripherally propagating injury discharge produced by damage to the peripheral nerve [28] and impulses traveling toward the periphery through the dorsal root reflex and axon reflex $[2,34]$. The algesic substances released in the periphery via the activation of nociceptors are involved in the production of inflammation, which leads to nociceptor sensitization $[1,8]$. Thus, it is possible that algesic substances released from the peripheral terminals of nociceptors may sensitize nearby nociceptors through a diffusion or reflex action. However, there has been much controversy over whether the sensitization of nociceptors spreads beyond the area of skin innervated by the injured or stimulated nerve [35-41]. This controversy is due, in part, to the use of different methods for nociceptor stimulation and different animal species for performing the experiments. Thus, conclusions have been drawn from conflicting and contradictory data.
The tetanic ES of decentralized L5 SN is a useful way to examine the involvement of peripherally propagating impulses and subsequently released algesic substances in pain hypersensitivity without considering the involvement of central sensitization by employing L5 DR-bearing rats, in which the central access of nerve impulses evoked by the ES of L5 SN is completely blocked. In the present study, the finding that tactile hypersensitivity developed in the center of the circle surrounded by the tori of the hind-paw (i.e., the midpoint of the L4 plantar dermatome) following the ES of the decentralized L5 SN indicates that skin hypersensitivity can occur near but outside the area of skin innervated by the stimulated L5 SN (Figures 1 and 2). Although neural mechanism of tissue hypersensitivity beyond the area of original injury, that is, secondary hyperalgesia, is generally considered to result from the sensitization of spinal dorsal horn neurons, a solid conclusion has not yet been drawn [42, 43]. The behavioral hypersensitivity observed in the present 


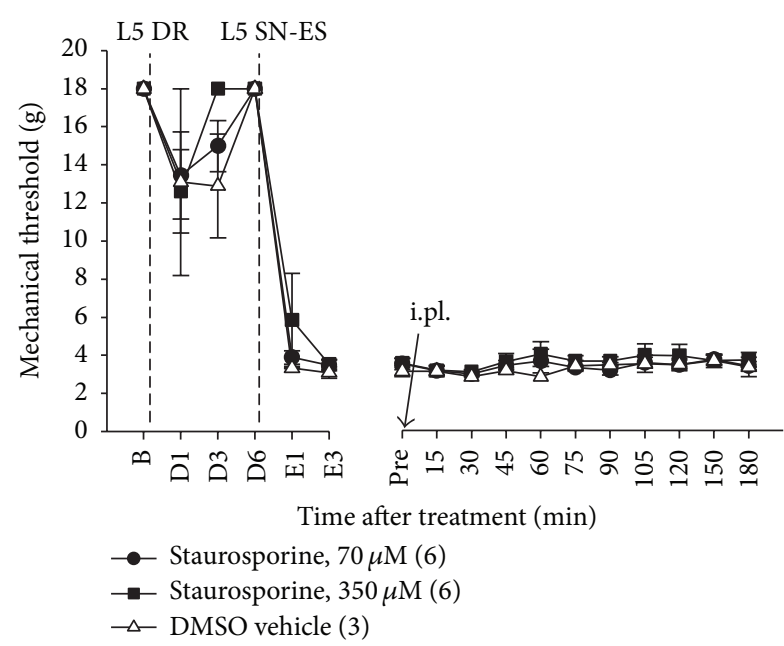

(a)

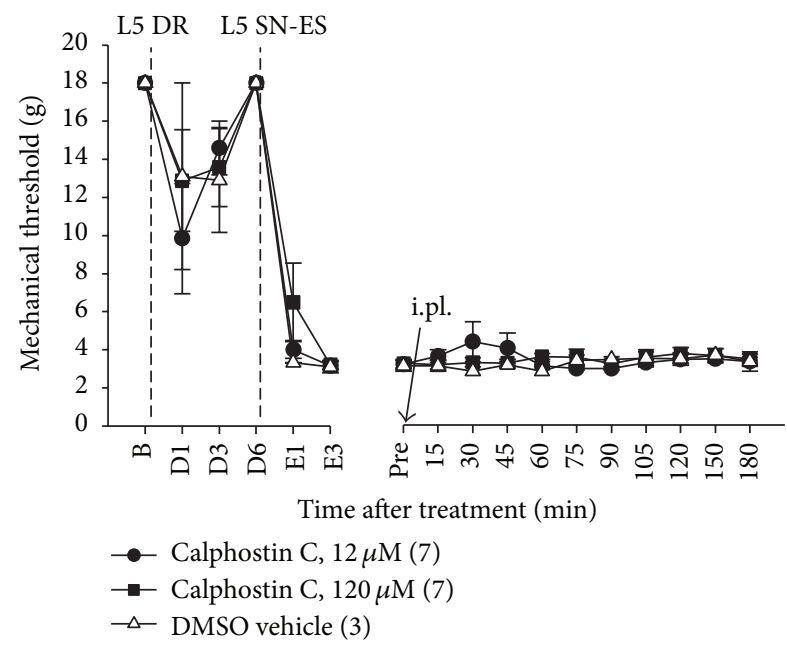

(b)

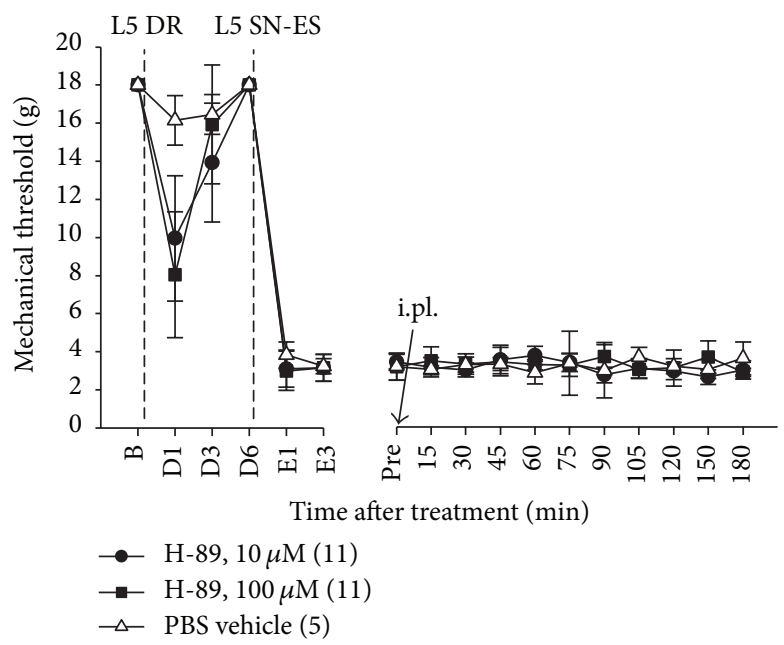

(c)

FIGURE 6: Effects of staurosporine, calphostin C, or H-89 posttreatment in the periphery on ES-induced PWT reduction. (a-c) In rats with L5 DR, an i.pl. injection of staurosporine $(70 \mu \mathrm{M})$, calphostin C $(12 \mu \mathrm{M})$, or H-89 $(10 \mu \mathrm{M})$ administered 3 days after the ES of L5 SN (L5 SNES) into the midpoint of the L4 plantar dermatome resulted in no significant difference in ES-induced decreased PWTs from vehicle-treated controls. No effects were observed with higher doses of each drug. The abbreviations B, D, E, and Pre are defined in Figure 4 . The values in parentheses indicate the number of animals used. The error bars represent the SEM.

study can be accounted for by the sensitization of nociceptors in the L4 plantar dermatome rather than central mechanisms. The importance of nociceptors supplying the L4 dermatome in retaining behavioral hypersensitivity seen in our model rats has previously been revealed by the complete blockage of such hypersensitivity after the elimination of C-nociceptors in the L4 SN with local capsaicin treatment [28].

One might argue that a prior L5 DR can alter the properties of central neurons to affect the tactile sensitivity in the L4 dermatome. The barrage of impulses elicited briefly at the time of the dorsal root transection, degeneration of proximal stump of transected dorsal root, and enlarged receptive fields of dorsal horn neurons in the L5 spinal segment might be involved. Indeed, a mild tactile hypersensitivity was induced in the L4 dermatome by the L5 DR. Although the underlying mechanisms involved are not clear, this mild hypersensitivity following the L5 DR completely vanished in less than a week and did not reappear thereafter throughout the 12week observation period [28], suggesting a return of response properties of affected neurons to the normal state before the ES experiment commenced. In the present study, observed plasma extravasation by tetanic ES of the L5 SN was not inducible by the L5 DR alone (data not shown), suggesting no noticeable effects of a prior L5 DR on response properties of nociceptors in the periphery. Thus, it is possible to assume that the tactile hypersensitivity in the L4 dermatome following the ES of the decentralized L5 SN and subsequent pharmacological modulation would not be confounded by a prior L5 DR.

4.2. Importance of NMDARs in Antidromic ES-Induced Hypersensitivity. The importance of the peripheral Glu and 


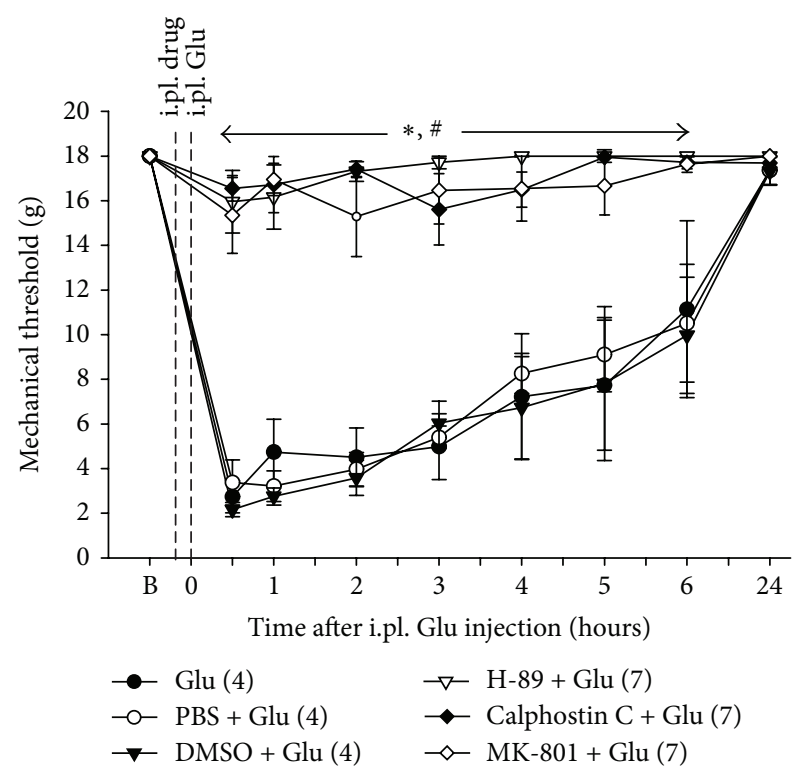

FIGURE 7: Effects of MK-801, calphostin C, or H-89 pretreatment in the periphery on Glu-induced PWT reduction. In naive rats, an i.pl. injection of Glu $(1 \mu \mathrm{M})$ into the midpoint of the L4 plantar dermatome resulted in a significant decrease in PWTs that lasted approximately $6 \mathrm{~h}$ compared with the baseline. Animals administered an i.pl. injection of MK-801 (20 nM), calphostin C $(12 \mu \mathrm{M})$, or $\mathrm{H}-89(10 \mu \mathrm{M}) 10 \mathrm{~min}$ prior to the i.pl. injection of Glu at the same site exhibited a significant increase in Glu-induced decreased PWTs compared with vehicle-pretreated controls. B denotes the baseline before the i.pl. injections of the test substances. The values in parentheses indicate the number of animals used. The error bars represent the SEM. ${ }^{*} P<0.05$ versus vehicle group. ${ }^{*} P<0.05$ versus baseline.

its receptors in developing tactile hyperalgesia has been acknowledged. The peripheral inhibition or activation of specific GluRs has been shown to attenuate enhanced tactile sensitivity in various experimental animal models of pain, for example, inflammatory $[17,19,21,22]$ and neuropathic $[15,23]$ pain, which are believed to be mediated at least in part by the increased sensitivity of nociceptors to tactile stimuli $[44,45]$. In the present study, the local blockade of NMDARs or group-I mGluRs or the local activation of group-II mGluRs in the L4 dermatome completely blocked the decentralized L5-SN-ES-induced tactile hypersensitivity (Figure 3), and an NMDAR antagonist administered locally into the L4 dermatome reversed such already established hypersensitivity (Figure 4). These findings suggest the expansion of the area of mechanically hypersensitive skin to the L4 dermatome, which can be accounted for by the spread of nociceptor sensitization through the diffusion of Glu into the L 4 dermatome from the release site in the L5 dermatome.

It is well known that NMDARs play a key role in the central sensitization of spinal nociceptive neurons [4648]. In addition, the data in the present study indicate the importance of peripheral NMDARs but not AMPA/kainate receptors in the induction and maintenance of behavioral hypersensitivity (Figures 3 and 4), in accordance with a previous report in which the local inhibition of peripheral
NMDARs in humans prevented the development of secondary hyperalgesia by a peripheral action [43]. Our data also revealed that the behavioral hypersensitivity could be blocked by the activation of peripheral group-II mGluRs, which suggests a desensitization of peripheral nociceptors via the activation of group-II mGluRs. A previous study has shown that group-I mGluRs are coexpressed with groupII mGluRs in peripheral afferent neurons [49]. In addition, the intrathecal administration of group-I mGluR agonists produces hyperalgesia [50], and the activation of groupI mGluRs results in the hyperexcitability of spinal dorsal horn neurons [51]. Thus, the coactivation of group-I and -II mGluRs by Glu may produce no changes in the excitability of nociceptors and subsequent behavioral sensitivity. We have previously shown that the local administration of groupI mGluR antagonists and group-II mGluR agonists in the periphery each hinders the initiation of tactile hypersensitivity in our modified neuropathic pain model whereas a nonselective antagonist of mGluR produces no such effect on hyperalgesia [15]. Although the coactivation of group-I and group-II mGluRs by Glu is less likely to participate in nociceptor sensitization and behavioral hypersensitivity, the use of substances that selectively block group-I mGluRs or selectively activate group-II mGluRs together with those that block NMDARs may prevent pain hypersensitivity related to nociceptor sensitization in the periphery.

\subsection{Involvement of PKA and PKC in Antidromic ES-Induced} Hypersensitivity. Some signal transduction receptors essential for sensing noxious mechanical stimuli have been identified in sensory neurons, which include the transient receptor potential Vanilloid 1 and Ankyrin 1 (TRPV1 and TRPA1) and piezo-type mechanosensitive cation channels (Piezo). For example, TRPA1 which is sensitized through various intracellular kinases such as PKA and phospholipase C (PLC) by inflammatory mediators, including bradykinin and prostaglandins, plays a critical role in tactile hyperalgesia [5255].

We demonstrated in the present study that antidromic nerve stimulation-induced tactile hypersensitivity was blocked by pretreatment with inhibitors of PKA and PKC in the periphery (Figure 5), which indicates the involvement of activated peripheral PKA and PKC in the initiation of tactile hypersensitivity. The tactile hypersensitivity observed in our study can be considered to be the result of the PKAand PKC-dependent sensitization of nociceptors to tactile stimuli mediated by peripheral Glu. There are several ways to account for such nociceptor sensitization. First, peripheral Glu activates adjacent nociceptors to release neuropeptides that attract and activate immune cells to release inflammatory mediators, resulting in nociceptor sensitization through protein kinase-dependent activation of transduction receptors as described above. Indeed, there are numerous pieces of evidence implying that SP and CGRP released from the peripheral terminals of primary sensory neurons induce nociceptor sensitization through the stimulation of immune cells to release inflammatory mediators [56-61]. Piezo2 on sensory neurons also can be involved in mediating 
inflammatory mediator-induced nociceptor sensitization. The potentiation of the activity of piezo2 through PKA and PKC has been found to mediate bradykinin-induced tactile hyperalgesia $[62,63]$. Second, peripheral Glu, which binds to NMDARs on the nearby nociceptors, can sensitize these nociceptors through the intracellular protein kinasedependent activation of transduction receptors. It has been shown that NMDA-induced tactile hyperalgesia is mediated by the activation of TRPV1 through CaMKII and PKC signaling cascades in trigeminal ganglion neurons [26, 27]. Third, the potentiation of NMDAR activity by mGluRs in sensory neurons via intracellular signaling pathways can be involved in NMDAR-mediated sensitization of nociceptors. Several pieces of evidence support this possibility. The activation of group-I mGluRs facilitates NMDAR-mediated responses in spinal dorsal horn neurons in the inflammatory hyperalgesic state [51]. The enhanced nociceptive processing in amygdala neurons by the activation of group-I mGluRs is mediated by reactive oxygen species (ROS) that activate PKA and ERK $[64,65]$. ROS are known to serve as important intracellular signaling molecules in peripheral and central pain mechanisms [66-69].

Our findings that tactile hypersensitivity induced by antidromic stimulation of L5 SN or by i.pl. Glu injected into the L4 dermatome was blocked by pretreatment with an NMDAR antagonist and both PKA and PKC inhibitors in the L4 dermatome (Figures 3, 5, and 7) strongly support the idea that peripherally released Glu sensitizes nearby nociceptors through PKA- and PKC-dependent mechanisms mediated by NMDAR activation to induce pain hypersensitivity. Although, in the present study, we have not attempted to determine specific neuronal cell types involved in peripheral Glu- or ES-induced pain hypersensitivity and PKA/PKC expression patterns, unmyelinated C-nociceptors are likely critically involved in this pathway. Most of all, capsaicin sensitive afferents are clearly required for ES-induced mechanical hypersensitivity [28]. Also, the fact that candidate molecules responsible for the Glu- or inflammation-induced mechanical hypersensitivity via the PKA or PKC signaling pathway (e.g., TRPV1 [26, 27], TRPA1 [54, 55], and TTX-resistant sodium channels $[70,71])$ are preferentially expressed on Cnociceptors supports this notion. Nevertheless, further study is warranted to confirm this possibility.

The already established tactile hypersensitivity following antidromic nerve stimulation was partially reversed by the blockade of NMDARs but not AMPA/kainate receptors, group-I mGluR, PKA, or PKC (Figures 4 and 6), which suggests that hypersensitivity is partly maintained by NMDAR activation. These results raise the possibility of the involvement of other NMDAR-mediated signaling molecules, such as ROS, PLC, and ERK, various transduction receptors, and other types of mGluRs in the maintenance of this hypersensitivity.

\section{Conclusions}

Our data suggest that peripheral Glu released by the antidromic stimulation of the decentralized $\mathrm{SN}$ results in the expansion of the area of tactile hyperalgesia via the spread of nociceptor sensitization, which is mediated by the diffusion of Glu into the skin near the release site. Our data also imply that intracellular PKA- and PKC-dependent mechanisms mediated mainly by NMDAR activation are involved in Glu-induced nociceptor sensitization and subsequent tactile hypersensitivity.

\section{Conflict of Interests}

The authors declare that there is no conflict of interests regarding the publication of this paper.

\section{Acknowledgments}

This work was supported by the Korea Health technology R\&D Project funded by the Ministry of Health \& Welfare (A120254) and the Bio \& Medical Technology Program through the NRF funded by the Ministry of Education, Science and Technology (2012M3A9C7050126 and 2012M3A9B4028631), Republic of Korea.

\section{References}

[1] P. Holzer, "Local effector functions of capsaicin-sensitive sensory nerve endings: involvement of tachykinins, calcitonin gene-related peptide and other neuropeptides," Neuroscience, vol. 24, no. 3, pp. 739-768, 1988.

[2] C. A. Maggi and A. Meli, "The sensory-efferent function of capsaicin-sensitive sensory neurons," General Pharmacology, vol. 19, no. 1, pp. 1-43, 1988.

[3] H. Sann and F.-K. Pierau, "Efferent functions of C-fiber nociceptors," Zeitschrift für Rheumatologie, vol. 57, supplement 2, pp. 8-13, 1998.

[4] J. deGroot, S. Zhou, and S. M. Carlton, "Peripheral glutamate release in the hindpaw following low and high intensity sciatic stimulation," NeuroReport, vol. 11, no. 3, pp. 497-502, 2000.

[5] C. A. Maggi, "Tachykinins and calcitonin gene-related peptide (CGRP) as co-transmitters released from peripheral endings of sensory nerves," Progress in Neurobiology, vol. 45, no. 1, pp. 1-98, 1995.

[6] N. Yonehara and M. Yoshimura, "Influence of painful chronic neuropathy on neurogenic inflammation," Pain, vol. 92, no. 1-2, pp. 259-265, 2001.

[7] J. M. Lundberg, A. Franco-Cereceda, K. Alving, P. Delay-Goyet, and Y.-P. Lou, "Release of calcitonin gene-related peptide from sensory neurons," Annals of the New York Academy of Sciences, vol. 657, pp. 187-193, 1992.

[8] J. D. Richardson and M. R. Vasko, "Cellular mechanisms of neurogenic inflammation," Journal of Pharmacology and Experimental Therapeutics, vol. 302, no. 3, pp. 839-845, 2002.

[9] G. Bhave, F. Karim, S. M. Carlton, and R. W. Gereau IV, "Peripheral group I metabotropic glutamate receptors modulate nociception in mice," Nature Neuroscience, vol. 4, no. 4, pp. 417423, 2001.

[10] S. M. Carlton, G. L. Hargett, and R. E. Coggeshall, "Localization and activation of glutamate receptors in unmyelinated axons of rat glabrous skin," Neuroscience Letters, vol. 197, no. 1, pp. 25-28, 1995. 
[11] S. M. Carlton, G. L. Hargett, and R. E. Coggeshall, "Localization of metabotropic glutamate receptors $2 / 3$ on primary afferent axons in the rat," Neuroscience, vol. 105, no. 4, pp. 957-969, 2001.

[12] R. E. Coggeshall and S. M. Carlton, "Ultrastructural analysis of NMDA, AMPA, and kainate receptors on unmyelinated and myelinated axons in the periphery," Journal of Comparative Neurology, vol. 391, no. 1, pp. 78-86, 1998.

[13] K. Walker, A. Reeve, M. Bowes et al., "mGlu5 receptors and nociceptive function II. mGlu5 receptors functionally expressed on peripheral sensory neurones mediate inflammatory hyperalgesia," Neuropharmacology, vol. 40, no. 1, pp. 10-19, 2001.

[14] S. Zhou, L. Bonasera, and S. M. Carlton, "Peripheral administration of NMDA, AMPA or KA results in pain behaviors in rats," NeuroReport, vol. 7, no. 4, pp. 895-900, 1996.

[15] J. H. Jang, D.-W. Kim, T. Sang Nam, K. Se Paik, and J. W. Leem, "Peripheral glutamate receptors contribute to mechanical hyperalgesia in a neuropathic pain model of the rat," Neuroscience, vol. 128, no. 1, pp. 169-176, 2004.

[16] Y.-H. Jin, M. Takemura, A. Furuyama, and N. Yonehara, "Peripheral glutamate receptors are required for hyperalgesia induced by capsaicin," Pain Research and Treatment, vol. 2012, Article ID 915706, 8 pages, 2012.

[17] H.-S. Chen, F. Qu, X. He, S.-M. Kang, D. Liao, and S.-J. $\mathrm{Lu}$, "Differential roles of peripheral metabotropic glutamate receptors in bee venom-induced nociception and inflammation in conscious rats," Journal of Pain, vol. 11, no. 4, pp. 321-329, 2010.

[18] E. M. Davidson, R. E. Coggeshall, and S. M. Carlton, "Peripheral NMDA and non-NMDA glutamate receptors contribute to nociceptive behaviors in the rat formalin test," Neuroreport, vol. 8, no. 4, pp. 941-946, 1997.

[19] H.-J. You, J. Chen, C. D. Morch, and L. Arendt-Nielsen, "Differential effect of peripheral glutamate (NMDA, non-NMDA) receptor antagonists on bee venom-induced spontaneous nociception and sensitization," Brain Research Bulletin, vol. 58, no. 6, pp. 561-567, 2002.

[20] D. L. Jackson, C. B. Graff, J. D. Richardson, and K. M. Hargreaves, "Glutamate participates in the peripheral modulation of thermal hyperalgesia in rats," European Journal of Pharmacology, vol. 284, no. 3, pp. 321-325, 1995.

[21] J. Du, S. Zhou, R. E. Coggeshall, and S. M. Carlton, "N-Methyl$\mathrm{D}$-aspartate-induced excitation and sensitization of normal and inflamed nociceptors," Neuroscience, vol. 118, no. 2, pp. 547-562, 2003.

[22] C. Y. Jung, S. Y. Lee, H. S. Choi et al., "Participation of peripheral group I and II metabotropic glutamate receptors in the development or maintenance of IL- $1 \beta$-induced mechanical allodynia in the orofacial area of conscious rats," Neuroscience Letters, vol. 409, no. 3, pp. 173-178, 2006.

[23] A. Dogrul, M. H. Ossipov, J. Lai, T. P. Malan Jr., and F. Porreca, "Peripheral and spinal antihyperalgesic activity of SIB-1757, a metabotropic glutamate receptor $\left(\mathrm{mGLUR}_{5}\right)$ antagonist, in experimental neuropathic pain in rats," Neuroscience Letters, vol. 292, no. 2, pp. 115-118, 2000.

[24] H. J. Lee, H. S. Choi, J. S. Ju et al., "Peripheral mGluR5 antagonist attenuated craniofacial muscle pain and inflammation but not mGluR1 antagonist in lightly anesthetized rats," Brain Research Bulletin, vol. 70, no. 4-6, pp. 378-385, 2006.

[25] J. Du, M. Koltzenburg, and S. M. Carlton, "Glutamate-induced excitation and sensitization of nociceptors in rat glabrous skin," Pain, vol. 89, no. 2-3, pp. 187-198, 2001.
[26] J. Lee, M.-K. Chung, and J. Y. Ro, "Activation of NMDA receptors leads to phosphorylation of TRPV1 S800 by protein kinase $\mathrm{C}$ and A-Kinase anchoring protein 150 in rat trigeminal ganglia," Biochemical and Biophysical Research Communications, vol. 424, no. 2, pp. 358-363, 2012.

[27] J. Lee, J. L. Saloman, G. Weiland, Q.-S. Auh, M.-K. Chung, and J. Y. Ro, "Functional interactions between NMDA receptors and TRPV1 in trigeminal sensory neurons mediate mechanical hyperalgesia in the rat masseter muscle," Pain, vol. 153, no. 7, pp. 1514-1524, 2012.

[28] J. H. Jang, B. H. Lee, T. S. Nam, J. W. Kim, D. W. Kim, and J. W. Leem, "Peripheral contributions to the mechanical hyperalgesia following a lumbar 5 spinal nerve lesion in rats," Neuroscience, vol. 165, no. 1, pp. 221-232, 2010.

[29] Y. Takahashi, Y. Nakajima, and T. Sakamoto, "Dermatome mapping in the rat hindlimb by electrical stimulation of the spinal nerves," Neuroscience Letters, vol. 168, no. 1-2, pp. 85-88, 1994.

[30] T. Ushida, T. Tani, M. Kawasaki, O. Iwatsu, and H. Yamamoto, "Peripheral administration of an N-methyl-D-aspartate receptor antagonist (MK-801) changes dorsal horn neuronal responses in rats," Neuroscience Letters, vol. 260, no. 2, pp. 89-92, 1999.

[31] Y.-H. Chun, D. Frank, J.-S. Lee, Y. Zhang, Q.-S. Auh, and J. Y. Ro, "Peripheral AMPA receptors contribute to muscle nociception and c-fos activation," Neuroscience Research, vol. 62, no. 2, pp. 97-104, 2008.

[32] W. J. Dixon, "Efficient analysis of experimental observations," Annual Review of Pharmacology and Toxicology, vol. 20, no. 1, pp. 441-462, 1980.

[33] S. R. Chaplan, F. W. Bach, J. W. Pogrel, J. M. Chung, and T. L. Yaksh, "Quantitative assessment of tactile allodynia in the rat paw," Journal of Neuroscience Methods, vol. 53, no. 1, pp. 55-63, 1994.

[34] W. D. Willis Jr., "Dorsal root potentials and dorsal root reflexes: a double-edged sword," Experimental Brain Research, vol. 124, no. 4, pp. 395-421, 1999.

[35] L. A. Chahl and R. J. Ladd, "Local oedema and general excitation of cutaneous sensory receptors produced by electrical stimulation of the saphenous nerve in the rat," Pain, vol. 2, no. 1, pp. 25-34, 1976.

[36] M. Fitzgerald, "The spread of sensitization of polymodal nociceptors in the rabbit from nearby injury and by antidromic nerve stimulation," Journal of Physiology, vol. 297, pp. 207-216, 1979.

[37] D. Li, Y. Ren, X. Xu, X. Zou, L. Fang, and Q. Lin, "Sensitization of primary afferent nociceptors induced by intradermal capsaicin involves the peripheral release of calcitonin gene-related Peptide driven by dorsal root reflexes," Journal of Pain, vol. 9, no. 12, pp. 1155-1168, 2008.

[38] P. Panopoulos, B. Gazelius, and L. Olgart, "Alterations in intradental nerve responsiveness induced by electrical stimulation of the inferior alveolar nerve in the cat," Acta Physiologica Scandinavica, vol. 118, no. 515, pp. 37-43, 1983.

[39] R. A. Meyer, J. N. Campbell, and S. N. Raja, "Antidromic nerve stimulation in monkey does not sensitize unmyelinated nociceptors to heat," Brain Research, vol. 441, no. 1-2, pp. 168$172,1988$.

[40] P. W. Reeh, L. Kocher, and S. Jung, "Does neurogenic inflammation alter the sensitivity of unmyelinated nociceptors in the rat?” Brain Research, vol. 384, no. 1, pp. 42-50, 1986. 
[41] M. Schmelz, R. Schmidt, M. Ringkamp, C. Forster, H. O. Handwerker, and H. E. Torebjörk, "Limitation of sensitization to injured parts of receptive fields in human skin Cnociceptors," Experimental Brain Research, vol. 109, no. 1, pp. 141-147, 1996.

[42] R.-D. Treede, R. A. Meyer, S. N. Raja, and J. N. Campbell, "Peripheral and central mechanisms of cutaneous hyperalgesia," Progress in Neurobiology, vol. 38, no. 4, pp. 397-421, 1992.

[43] T. Warncke, E. Jørum, and A. Stubhaug, "Local treatment with the $N$-methyl-D-aspartate receptor antagonist ketamine, inhibit development of secondary hyperalgesia in man by a peripheral action," Neuroscience Letters, vol. 227, no. 1, pp. 1-4, 1997.

[44] B. Shim, D.-W. Kim, B. H. Kim, T. S. Nam, J. W. Leem, and J. M. Chung, "Mechanical and heat sensitization of cutaneous nociceptors in rats with experimental peripheral neuropathy," Neuroscience, vol. 132, no. 1, pp. 193-201, 2005.

[45] D. Andrew and J. D. Greenspan, "Mechanical and heat sensitization of cutaneous nociceptors after peripheral inflammation in the rat," Journal of Neurophysiology, vol. 82, no. 5, pp. 26492656, 1999.

[46] C. J. Woolf and S. W. N. Thompson, "The induction and maintenance of central sensitization is dependent on N-methyl-Daspartic acid receptor activation; implications for the treatment of post-injury pain hypersensitivity states," Pain, vol. 44, no. 3, pp. 293-299, 1991.

[47] A. H. Dickenson and A. F. Sullivan, "Evidence for a role of the NMDA receptor in the frequency dependent potentiation of deep rat dorsal horn nociceptive neurones following $\mathrm{C}$ fibre stimulatio," Neuropharmacology, vol. 26, no. 8, pp. 1235-1238, 1987.

[48] T. J. Coderre and R. Melzack, "The contribution of excitatory amino acids to central sensitization and persistent nociception after formalin-induced tissue injury," Journal of Neuroscience, vol. 12, no. 9, pp. 3665-3670, 1992.

[49] S. M. Carlton and G. L. Hargett, "Colocalization of metabotropic glutamate receptors in rat dorsal root ganglion cells," Journal of Comparative Neurology, vol. 501, no. 5, pp. 780-789, 2007.

[50] B. H. Gabra, F. K. Kessler, J. K. Ritter, W. L. Dewey, and F. L. Smith, "Decrease in $N$-methyl-D-aspartic acid receptor-NR2B subunit levels by intrathecal short-hairpin RNA blocks group I metabotropic glutamate receptor-mediated hyperalgesia," Journal of Pharmacology and Experimental Therapeutics, vol. 322, no. 1, pp. 186-194, 2007.

[51] K. Yang, K. Takeuchi, F. Wei, R. Dubner, and K. Ren, "Activation of group I mGlu receptors contributes to facilitation of NMDA receptor membrane current in spinal dorsal horn neurons after hind paw inflammation in rats," European Journal of Pharmacology, vol. 670, no. 2-3, pp. 509-518, 2011.

[52] M. Petrus, A. M. Peier, M. Bandell et al., "A role of TRPA1 in mechanical hyperalgesia is revealed by pharmacological inhibition," Molecular Pain, vol. 3, article 40, 2007.

[53] K. Y. Kwan, A. J. Allchorne, M. A. Vollrath et al., “TRPA1 contributes to cold, mechanical, and chemical nociception but is not essential for hair-cell transduction," Neuron, vol. 50, no. 2, pp. 277-289, 2006.

[54] S. Wang, Y. Dai, T. Fukuoka et al., "Phospholipase C and protein kinase A mediate bradykinin sensitization of TRPA1: a molecular mechanism of inflammatory pain," Brain, vol. 131, no. 5, pp. 1241-1251, 2008.
[55] R. C. Lennertz, E. A. Kossyreva, A. K. Smith, and C. L. Stucky, "TRPA1 mediates mechanical sensitization in nociceptors during inflammation," PLoS ONE, vol. 7, no. 8, Article ID e43597, 2012.

[56] J. C. Ansel, J. R. Brown, D. G. Payan, and M. A. Brown, "Substance P selectively activates TNF-alpha gene expression in murine mast cells," The Journal of Immunology, vol. 150, no. 10, pp. 4478-4485, 1993.

[57] N. Mikami, H. Matsushita, T. Kato et al., "Calcitonin generelated peptide is an important regulator of cutaneous immunity: effect on dendritic cell and T cell functions," Journal of Immunology, vol. 186, no. 12, pp. 6886-6893, 2011.

[58] C. M. Bullock, P. Wookey, A. Bennett, A. Mobasheri, I. Dickerson, and S. Kelly, "Peripheral calcitonin gene-related peptide receptor activation and mechanical sensitization of the joint in rat models of osteoarthritis pain," Arthritis and Rheumatology, vol. 66, no. 8, pp. 2188-2200, 2014.

[59] S. Kelly, J. P. Dunham, F. Murray, S. Read, L. F. Donaldson, and S. N. Lawson, "Spontaneous firing in C-fibers and increased mechanical sensitivity in A-fibers of knee joint-associated mechanoreceptive primary afferent neurones during MIAinduced osteoarthritis in the rat," Osteoarthritis and Cartilage, vol. 20, no. 4, pp. 305-313, 2012.

[60] X.-C. Zhang, V. Kainz, R. Burstein, and D. Levy, “Tumor necrosis factor- $\alpha$ induces sensitization of meningeal nociceptors mediated via local COX and p38 MAP kinase actions," Pain, vol. 152, no. 1, pp. 140-149, 2011.

[61] H. Junger and L. S. Sorkin, "Nociceptive and inflammatory effects of subcutaneous TNF $\alpha$," Pain, vol. 85, no. 1-2, pp. 145$151,2000$.

[62] A. E. Dubin, M. Schmidt, J. Mathur et al., "Inflammatory signals enhance piezo2-mediated mechanosensitive currents," Cell Reports, vol. 2, no. 3, pp. 511-517, 2012.

[63] S. E. Kim, B. Coste, A. Chadha, B. Cook, and A. Patapoutian, "The role of Drosophila Piezo in mechanical nociception," Nature, vol. 483, no. 7388, pp. 209-212, 2012.

[64] G. Ji and V. Neugebauer, "Reactive oxygen species are involved in group I mGluR-mediated facilitation of nociceptive processing in amygdala neurons," Journal of Neurophysiology, vol. 104, no. 1, pp. 218-229, 2010.

[65] Z. Li, G. Ji, and V. Neugebauer, "Mitochondrial reactive oxygen species are activated by mGluR5 through IP3 and activate ERK and PKA to increase excitability of amygdala neurons and pain behavior," Journal of Neuroscience, vol. 31, no. 3, pp. 1114-1127, 2011.

[66] E. S. Schwartz, I. Lee, K. Chung, and J. M. Chung, "Oxidative stress in the spinal cord is an important contributor in capsaicin-induced mechanical secondary hyperalgesia in mice," Pain, vol. 138, no. 3, pp. 514-524, 2008.

[67] Z.-Q. Wang, F. Porreca, S. Cuzzocrea et al., "A newly identified role for superoxide in inflammatory pain," Journal of Pharmacology and Experimental Therapeutics, vol. 309, no. 3, pp. 869878, 2004.

[68] X. Gao, H. K. Kim, J. M. Chung, and K. Chung, "Reactive oxygen species (ROS) are involved in enhancement of NMDAreceptor phosphorylation in animal models of pain," Pain, vol. 131, no. 3, pp. 262-271, 2007.

[69] J. E. Keeble, J. V. Bodkin, L. Liang et al., "Hydrogen peroxide is a novel mediator of inflammatory hyperalgesia, acting via transient receptor potential vanilloid 1-dependent and independent mechanisms," Pain, vol. 141, no. 1-2, pp. 135-142, 2009. 
[70] M. S. Gold, J. D. Levine, and A. M. Correa, "Modulation of TTX-R I(Na) by PKC and PKA and their role in PGE2-induced sensitization of rat sensory neurons in vitro," The Journal of Neuroscience, vol. 18, no. 24, pp. 10345-10355, 1998.

[71] S. K. Joshi, J. P. Mikusa, G. Hernandez et al., "Involvement of the TTX-resistant sodium channel Nav 1.8 in inflammatory and neuropathic, but not post-operative, pain states," Pain, vol. 123, no. 1-2, pp. 75-82, 2006. 


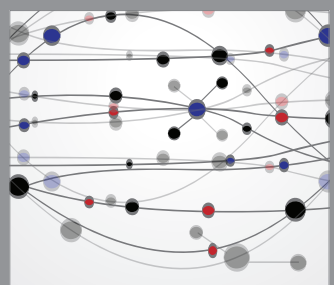

The Scientific World Journal
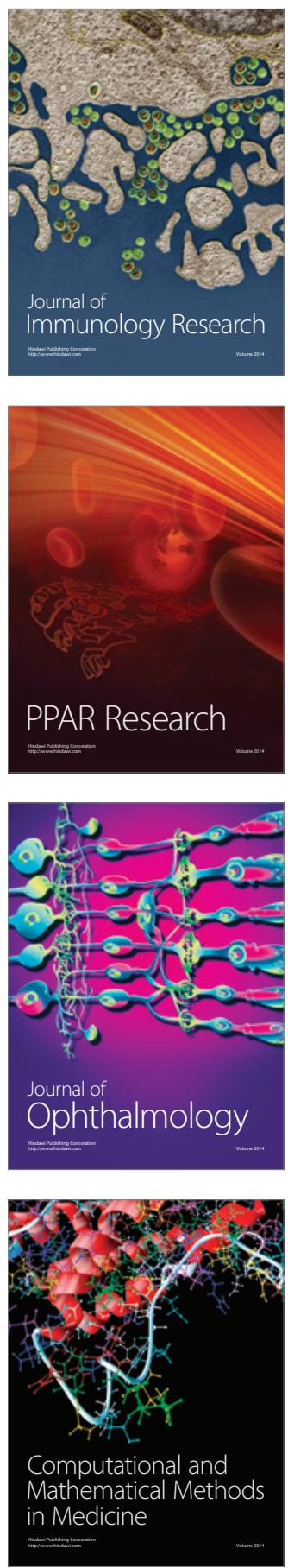

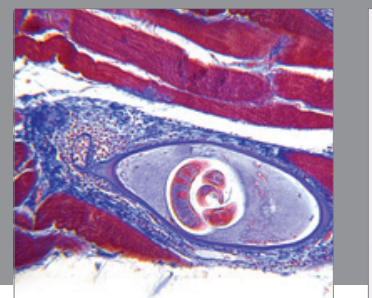

Gastroenterology

Research and Practice
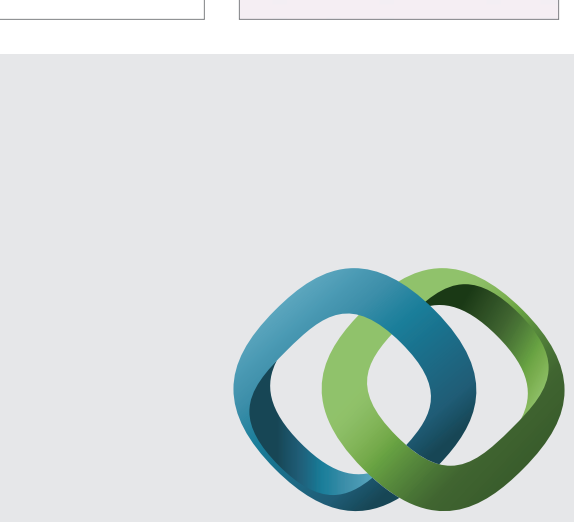

\section{Hindawi}

Submit your manuscripts at

http://www.hindawi.com
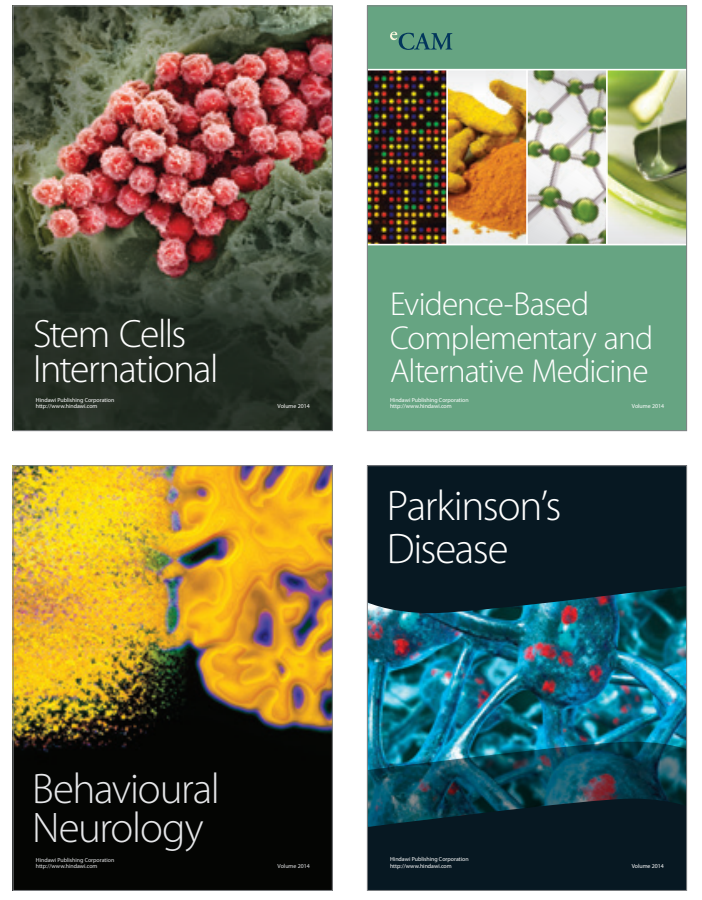
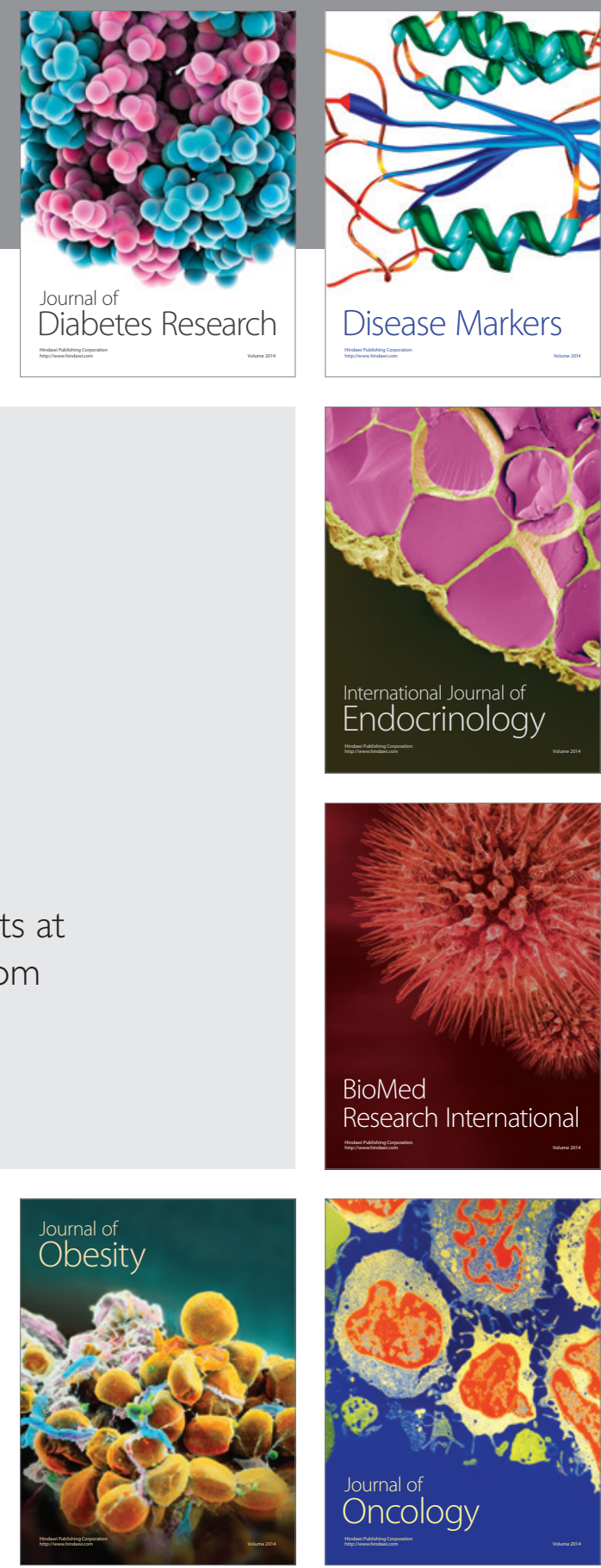

Disease Markers
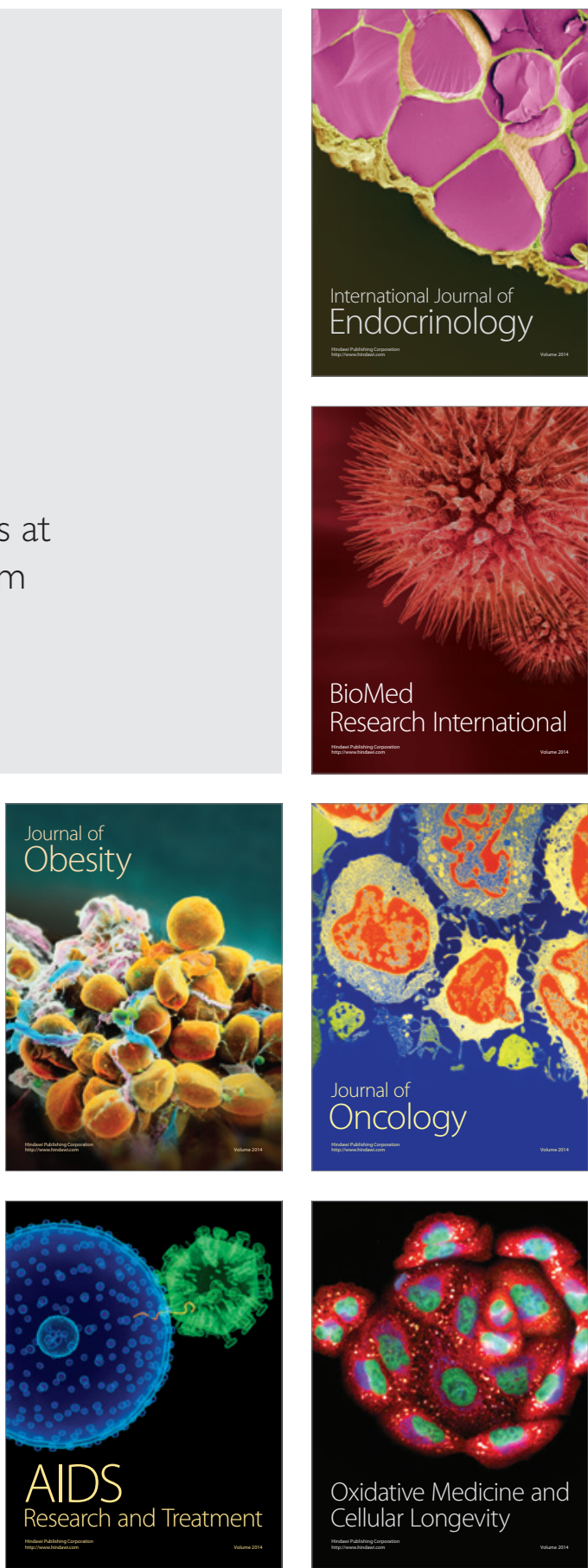